\title{
Identifying essential genes/reactions of the rice photorespiration by in silico model-based analysis
}

\author{
Meiyappan Lakshmanan ${ }^{1}$, Bijayalaxmi Mohanty ${ }^{1}$ and Dong-Yup Lee ${ }^{1,2^{*}}$
}

\begin{abstract}
Background: Photorespiration, a highly wasteful process of energy dissipation, depresses the productivity of C3 plants such as rice (Oryza sativa) under dry and hot conditions. Thus, it is highly required to understand the cellular physiology and relevant metabolic states under photorespiration using systems approaches, thereby devising strategies for improving rice production.

Findings: In silico model-driven gene deletion analysis was performed on photorespiring leaf cells under ambient and stressed environmental conditions using our central metabolic network of rice cells. As a result, we identified a number of essential genes for the cell growth across various functional pathways such as photorespiratory cycle, Calvin cycle, GS-GOGAT cycle and sucrose metabolism as well as certain inter-compartmental transporters, which are mostly in good agreement with previous experiments. Synthetic lethal (SL) screening was also performed to identify the pair of non-essential genes whose simultaneous deletion become lethal, revealing the existence of more than 220 pairs of SLs on rice central metabolism.

Conclusions: The gene deletion and synthetic lethal analyses highlighted the rigid nature of rice photosynthetic pathways and characterized functional interactions between central metabolic genes, respectively. The biological roles of such reported essential genes should be further explored to better understand the rice photorespiration in future.
\end{abstract}

Keywords: Rice metabolism; Photorespiration; Gene essentiality; Metabolic network model; Constraints-based flux analysis; Synthetic lethality

\section{Findings}

In silico model driven analysis of rice photorespiration Drought stress is one of the major environmental factors affecting the growth and development of rice due to high levels of photorespiration. Thus, in order to investigate how this abiotic stress affects the rice physiology via metabolic adaptations, it is essential to characterize the cellular behavior during photorespiration. The process is initiated by the oxygenase side reaction of the bifunctional ribulose-1,5-bisphosphate carboxylase/oxygenase (RuBisCO), producing equimolar amounts of 3-phosphoglycerate (3-PGA) and unwanted 2-phosphoglycolate (2-PG) for each molecule

\footnotetext{
*Correspondence: cheld@nus.edu.sg
'Department of Chemical and Biomolecular Engineering, National University

*Correspondence: cheld@nus.edu.sg
'Department of Chemical and Biomolecular Engineering, National University of Singapore, 4 Engineering Drive 4, Singapore 117576, Singapore ${ }^{2}$ Bioprocessing Technology Institute, Agency for Science, Technology and Research (A*STAR), 20 Biopolis Way, \#06-01, Centros, Singapore 138668, Singapore
}

of $\mathrm{O}_{2}$ fixed (Jordan and Ogren 1984). It is followed by the salvage of 2-PG into 3-PGA via photorespiratory pathway, requiring significant amount of cellular energy, i.e. ATP, in C3 plants such as rice (Wingler et al. 2000). The ratio of carboxylase/oxygenase reactions $\left(\mathrm{V}_{\mathrm{C}} /\right.$ $\mathrm{V}_{\mathrm{O}}$ ) is three under normal conditions (Heldt and Piechulla 2011), however, this ratio can drop even below one and may reach the compensation point $\left(\mathrm{V}_{\mathrm{C}} / \mathrm{V}_{\mathrm{O}}=0.5\right)$ at which the net $\mathrm{CO}_{2}$ uptake rate becomes zero under drought conditions (Heldt and Piechulla 2011). Therefore, the control of photorespiration has always been a main focus for improving rice productivity.

To date, a number of mutational studies have been performed in many C3 plants, mainly in Arabidopsis and barley, to understand the photorespiratory pathway, but identified only a handful of essential enzymes including serine-glyoxylate aminotransferase (SGAT), glycine decarboxylase (GDC), ferredoxin-dependent glutamate synthase (Fd-GOGAT) and glutamine synthase (GS) (Foyer et al. \\ 空 Springer}


2009) due to the limitations in mutant isolation process and the possible involvement of alternate pathways and genetic redundancy of the relevant enzymes (Reumann 2004; Timm et al. 2008). Thus, it is highly required to exploit more systematic approaches for improving our understanding of the rice photorespiration. In this regard, in silico metabolic modeling and analysis allow us to predict the cellular behaviour and metabolic states globally upon various environmental/genetic changes (Lewis et al. 2012). For example, in silico knock-out mutant studies and comparative analysis have provided the conditionally essential gene sets and corresponding functional modules under various growth conditions in E. coli and S. cerevisiae (Segre et al. 2005; Joyce et al. 2006). Similarly, in this work, we aim to elucidate the rice photorespiration under ambient and drought conditions by identifying essential genes using our recently reconstructed rice metabolic/regulatory network model which was validated with cell culture experiments (Lakshmanan et al. 2013).

\section{Identification of essential genes in rice photorespiration}

Both normal $\left(\mathrm{V}_{\mathrm{C}} / \mathrm{V}_{\mathrm{O}}=3\right)$ and stressed $\left(\mathrm{V}_{\mathrm{C}} / \mathrm{V}_{\mathrm{O}}=1\right)$ conditions during the rice photorespiration were simulated to evaluate the gene essentiality for cell growth by resorting to constraints-based flux analysis (see Method in Additional file 1). The results revealed about $60 \%$ of the reactions in the model were non-essential under both conditions while $25 \%$ were completely essential and distributed across various pathways of rice central metabolism as illustrated in Figure 1. Most of the essential genes were identified in photosynthetic pathways such as photorespiratory cycle (10 genes) and Calvin cycle (7 genes), indicating the rigidity of $\mathrm{CO}_{2}$ fixing mechanism in plants. Generally, these observations are in good agreement with the existing experimental evidences available on other plants such as Arabidopsis, pea, barley and maize (Table 1). The first enzyme of the photorespiratory pathway, phosphoglycolate phosphatase (PGLP), metabolizes the toxic 2-PG which may accumulate as a result of ribulose-1,5-bisphosphate (RuBP) oxygenase activity. If the 2-PG is not scavenged, it can inhibit the key glycolytic enzyme, triose phosphate isomerase (TPI), thereby disrupting photosynthesis even under ambient air (Somerville and Ogren 1979). Likewise, other photorespiratory enzymes such as glycolate oxidase (GOX) and SGAT are also essential for cell growth under both normal and stressed conditions by degrading the toxic metabolites, glycolate and glyoxylate, respectively (Zelitch et al. 2009; Wingler et al. 2000). Interestingly, serine hydroxymethyltransferase (mitochondrial) (SHM1: EC. 2.1.2.1), on the other hand, was found to be essential only under dry and hot conditions, which is highly consistent with the experiments by Voll et al. (2006), who reported the conditional viability of the SHM1 mutant of

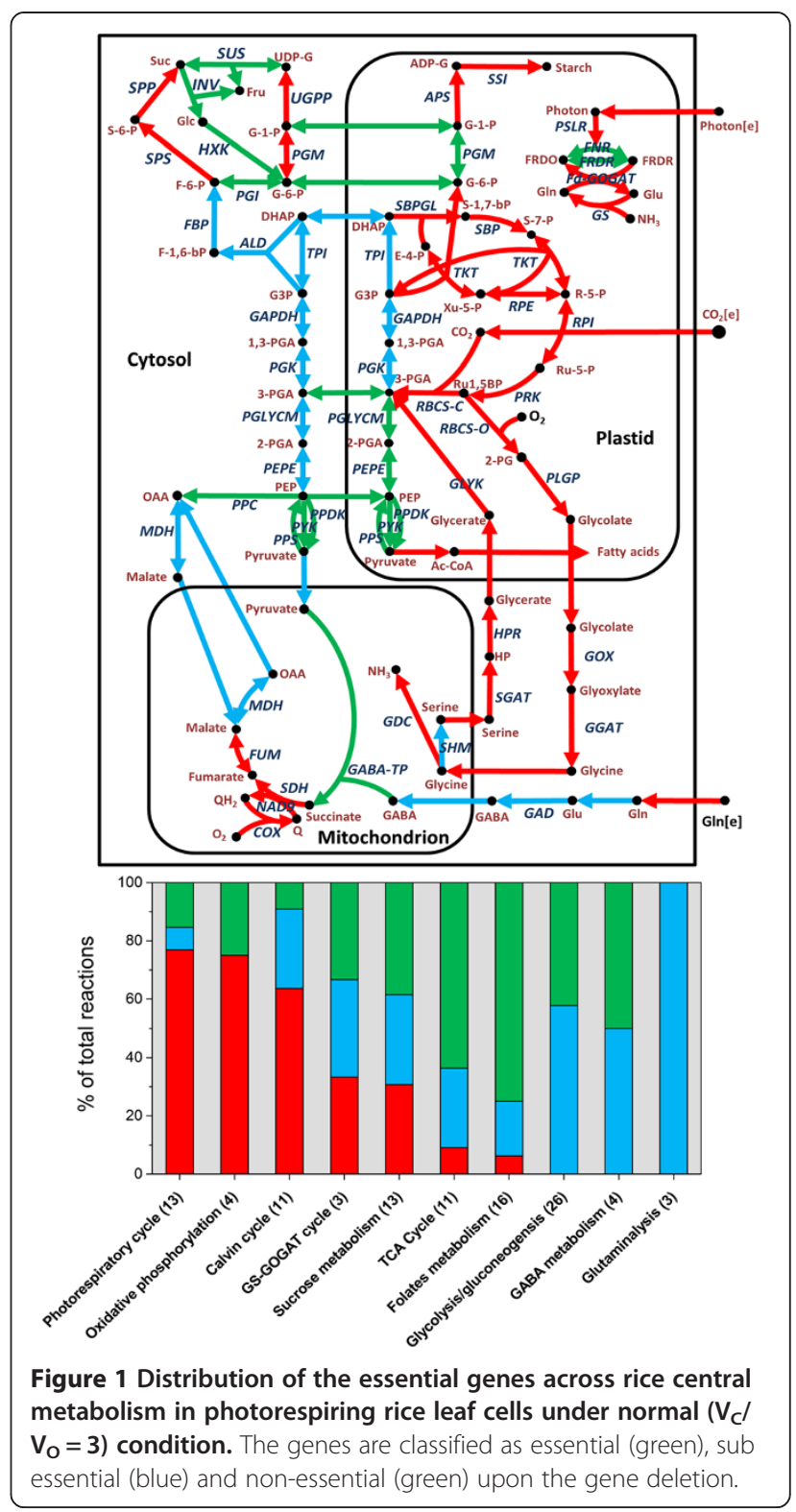

Arabidopsis thaliana. In order to further verify the results in rice, such predicted genes on Calvin cycle, photorespiratory pathway and GS-GOGAT cycle were compared with the essential genes of Arabidopsis and maize (Wang et al. 2012). Again, most of essential enzymes are common across all three plants, except PGLP with supporting experiments for our prediction (Somerville and Ogren 1979).

In addition to essential metabolic genes, we also analysed the dispensability of inter-compartmental metabolite transporters since the mechanism of photorespiration is quite intricate, involving three major organelles, chloroplast, mitochondrion and peroxisome. In this regard, we identified a number of essential inter-compartmental transporters including mitochondrial and plastidic malate/ 
Table 1 Comparison of essential genes/reactions in rice, Arabidopsis and maize during photorespiration

\begin{tabular}{|c|c|c|c|c|c|c|}
\hline \multirow[t]{2}{*}{ Enzyme } & \multirow{2}{*}{$\begin{array}{l}\text { EC } \\
\text { number }\end{array}$} & \multirow[t]{2}{*}{ Pathway } & \multirow{2}{*}{$\begin{array}{c}\text { Rice (C3) } \\
\text { (This study) }\end{array}$} & Arabidopsis (C3) & Maize (C4) & \multirow[t]{2}{*}{ References } \\
\hline & & & & \multicolumn{2}{|c|}{ Wang et al. (2012) } & \\
\hline RuBisCO & 4.1.1.39 & Calvin cycle & $\sqrt{ }$ & $\sqrt{ }$ & $\sqrt{ }$ & Sicher and Bunce (1997) \\
\hline PRK & 2.7.1.19 & Calvin cycle & $\sqrt{ }$ & $\sqrt{ }$ & $\sqrt{ }$ & Moll and Levine (1970) \\
\hline RPE & 5.3.1.6 & Calvin cycle & $\sqrt{ }$ & $\sqrt{ }$ & $\sqrt{ }$ & \\
\hline RPI & 5.1.3.1 & Calvin cycle & $\sqrt{ }$ & $\sqrt{ }$ & $\sqrt{ }$ & \\
\hline TKT & 2.2.1.1 & Calvin cycle & $\sqrt{ }$ & $\sqrt{ }$ & $\sqrt{ }$ & \\
\hline SBPase & 3.1.3.37 & Calvin cycle & $\sqrt{ }$ & NA & NA & Liu et al. (2012) \\
\hline PLGP & 3.1.3.18 & Photorespiratory cycle & $\sqrt{ }$ & $x$ & $x$ & Somerville and Ogren (1979) \\
\hline SHM & 2.1 .2 .1 & Photorespiratory cycle & $\sqrt{*}$ & NA & NA & Voll et al. (2006) \\
\hline GLYK & 2.7.1.31 & Photorespiratory cycle & $\sqrt{ }$ & NA & NA & Boldt et al. (2005) \\
\hline GDC & & Photorespiratory cycle & $\sqrt{ }$ & NA & NA & Wingler et al. (1997) \\
\hline Catalase & 1.11.1.6 & Photorespiratory cycle & $\sqrt{ }$ & NA & NA & \\
\hline GAL & 6.3 .1 .2 & Photorespiratory cycle & $\sqrt{ }$ & NA & NA & \\
\hline SGAT & 2.6.1.45 & Photorespiratory cycle & $\sqrt{ }$ & NA & NA & Wingler et al. (1999) \\
\hline GOX & 1.1.3.15 & Photorespiratory cycle & $\sqrt{ }$ & $\sqrt{ }$ & $\sqrt{ }$ & Zelitch et al. (2009) \\
\hline GS & 4.2.1.2 & GS-GOGAT cycle & $\sqrt{ }$ & NA & NA & Blackwell et al. (1987) \\
\hline Fd-GOGAT & 3.1.3.24 & GS-GOGAT cycle & $\sqrt{ }$ & NA & NA & Somerville and Ogren (1986) \\
\hline GLBE & 2.4.1.18 & Starch biosynthesis & $\sqrt{ }$ & $\sqrt{ }$ & $\sqrt{ }$ & \\
\hline PPC & 4.1.1.31 & & $x$ & $x$ & $\sqrt{ }$ & \\
\hline
\end{tabular}

$\sqrt{ }$ - essential gene; $X$ - non-essential gene; NA- essentiality not reported/investigated; ${ }^{*}$ - essential only under drought conditions.

fumarate/succinate redox shuttles in both conditions. Malate transporters play an essential role in transmitting the excess redox cofactors from plastid to mitochondria for their eventual utilization in oxidative phosphorylation while plastidic glycolate/glycerate transporter (PLGG) was reported as the core photorespiratory transporter (Pick et al. 2013). Additionally, a few unique mitochondrial transporters such as serine translocator, alpha-ketoglutarate/malate and glutamate/malate redox shuttles were identified to be essential only under stressed conditions, emphasizing their crucial roles in transporting the high fluxes of photorespiratory intermediates such as glycolate, glycerate, glutamate, oxoglutarate, glycine, and serine (Reumann and Weber 2006) (see Additional file 2 for the entire list of essential transporters).

From our analysis, it is evident that most of the photorespiratory enzymes including PGLP, GOX and SGAT are required for degrading the toxic metabolites and synthesizing signaling compounds such as $\mathrm{H}_{2} \mathrm{O}_{2}$ and glutathione (Wingler et al. 2000). Therefore, more carbohydrates and energy production via Calvin cycle toward the enhanced crop productivity can be achieved by increasing $\mathrm{CO}_{2}$ concentration around $\mathrm{RuBisCO}$ rather than blocking carbon fluxes through photorespiratory pathway.

\section{Synthetic lethality screening of non-essential gene pairs in rice photorespiration}

Besides identifying essential genes/reactions, we also newly screened the synthetic lethal (SL) gene pairs of rice central metabolism during photorespiration under normal and stressed conditions to better characterize the functional interactions between the non-essential genes (see Methods in Additional file 1). Note that SLs are pair of nonessential genes whose simultaneous removal can lead to zero growth (Suthers et al. 2009). Such lethality arises due to several reasons including interchangeable gene products with respect to an essential function (isozymes/isoforms), their existence in the same essential pathway or sharing of complementary essential function(s) (Suthers et al. 2009). Here, it should be noted that we excluded the intercompartmental transporters during SL screening since the deletion of most of the transporters coupled with metabolic genes resulted in no growth. A total of 226 and 229 SLs were identified in the normal and stressed conditions, respectively. Interestingly, of the total $226 \mathrm{SLs}$ the ferredoxin-NADP ${ }^{+}$reductase (FNR) in GS-GOGAT cycle, and the mitochondrial ATP synthase (ATPS) in oxidative phosphorylation were paired with 83 and 82 other genes of rice central metabolism, respectively. FNR is involved in reassimilating the ammonia released during photorespiration via Fd-GOGAT and maintaining the redox balance of plastids (Foyer et al. 2009) whereas ATPS is utilized to generate necessary energy for the cell growth via mitochondrial respiration (Lakshmanan et al. 2013). Several SLs also contained the isoforms of same enzymes across different compartments. Such examples include the cytosolic and plastidic isoforms of enolase, phosphoglycerate kinase, 
glyceraldehyde-3-phosphate dehydrogenase and triosephosphate isomerase, and the cytosolic and mitochondrial isoforms of malate dehydrogenase (see Additional file 2 for the entire list of SLs).

\section{Concluding remarks}

In the present study, we reported the essential genes and synthetic lethal gene pairs of rice central metabolism during photorespiration using in silico model-driven analysis. Our model simulations have unraveled several new essential genes of the photorespiratory metabolism, in addition to those which are reported earlier. However, it should be noted that gene essentiality results are condition-specific and sensitive to the model completeness. Therefore, the list of essential genes presented in the current study should be further confirmed with enhanced model predictability and subsequent experimental validations.

\section{Additional files}

Additional file 1: Details of the methods used in the study.

Additional file 2: List of essential inter-compartmental transporters and list of synthetic lethal gene pairs of rice central metabolism under normal and stressed photorespiration.

\section{Abbreviations}

2-PG: 2-phosphoglycolate; 3-PGA: 3-phosphoglycerate; ATPS: ATP synthase; CAT: Catalase; Fd-GOGAT: Ferredoxin-dependent glutamate synthase; FNR: Ferredoxin-NADP + reductase (plastidic); GAL: Glutamate - ammonia ligase; GDC: Glycine decarboxylase; GLBE: 1,4-alpha-glucan branching enzyme; GLYK: Glycerate kinase; GOX: Glycolate oxidase; GS: Glutamine synthase; HPR: Hydroxypyruvate reductase; PGLP: Phosphoglycolate phosphatase; PLGG: Plastidic glycolate/glycerate translocator; PRK: Phosphoribulokinase; RPE: Ribulose phosphate 3-epimerase; RPI: Ribulose phosphate 3-isomerase; RuBisCO: D-ribulose-1,5-bisphosphate carboxylase/oxygenase; RuBP: D-ribulose-1,5-bisphosphate;

SBPase: Sedoheptulose-bisphosphatase; SGAT: Serine-glyoxylate aminotransferase; SHM: Serine hydroxymethyltransferase; SL: Synthetic lethal; SPP: Sucrose-phosphatase; TKT: Transketolase; TPI: Triose phosphate isomerase.

\section{Competing interests}

The authors declare that they have no competing interests.

\section{Authors' contributions}

$M L, B M$ and DYL conceived and designed the study. ML carried out all simulation and analysis. ML, BM and DYL wrote the manuscript. All authors read and approved the final manuscript.

\section{Acknowledgements}

This work was supported by the National University of Singapore, Biomedical Research Council of A*STAR (Agency for Science, Technology and Research), Singapore, and a grant from the Next-Generation BioGreen 21 Program (SSAC, No. PJ009520), Rural Development Administration, Republic of Korea.

Received: 9 May 2013 Accepted: 30 July 2013

Published: 13 August 2013

\section{References}

Blackwell RD, Murray AJS, Lea PJ (1987) Inhibition of photosynthesis in barley with decreased levels of chloroplastic glutamine synthetase activity. J Exp Bot 38(11):1799-1809. doi:10.1093/jxb/38.11.1799
Boldt R, Edner C, Kolukisaoglu U, Hagemann M, Weckwerth W, Wienkoop S, Morgenthal K, Bauwe H (2005) D-GLYCERATE 3-KINASE, the last unknown enzyme in the photorespiratory cycle in Arabidopsis, belongs to a novel kinase family. The Plant cell 17(8):2413-2420. doi:10.1105/tpc.105.033993

Foyer CH, Bloom AJ, Queval G, Noctor G (2009) Photorespiratory metabolism: genes, mutants, energetics, and redox signaling. Annu Rev Plant Biol 60:455-484. doi:10.1146/annurev.arplant.043008.091948

Heldt HW, Piechulla B (2011) In the photorespiratory pathway phosphoglycolate formed by the oxygenase activity of RubisCo is recycled, Plant biochemistry. Academic, London, pp 193-209

Jordan DB, Ogren WL (1984) The $\mathrm{CO}_{2} / \mathrm{O}_{2}$ specificity of ribulose 1, 5-bisphosphate carboxylase/oxygenase. Planta 1618(1):308-313. doi:10.1007/BF00398720

Joyce AR, Reed JL, White A, Edwards R, Osterman A, Baba T, Mori H, Lesely SA, Palsson BO, Agarwalla S (2006) Experimental and computational assessment of conditionally essential genes in Escherichia coli. J Bacteriol 188(23):8259-8271. doi:10.1128/JB.00740-06

Lakshmanan M, Zhang Z, Mohanty B, Kwon J-Y, Choi H-Y, Nam H-J, Kim D-I, Lee D-Y (2013) Elucidating the rice cells metabolism under flooding and drought stresses using flux-based modelling and analysis. Plant Physiol 162(4):2140-2150. doi:10.1104/pp.113.220178

Lewis NE, Nagarajan H, Palsson BO (2012) Constraining the metabolic genotypephenotype relationship using a phylogeny of in silico methods. Nat Rev Microbiol 10(4):291-305. doi:10.1038/Nrmicro2737

Liu XL, Yu HD, Guan Y, Li JK, Guo FQ (2012) Carbonylation and loss-of-function analyses of SBPase reveal its metabolic interface role in oxidative stress, carbon assimilation, and multiple aspects of growth and development in Arabidopsis. Mol Plant 5(5):1082-1099. doi:10.1093/Mp/Sss012

Moll B, Levine RP (1970) Characterization of a photosynthetic mutant strain of Chlamydomonas-Reinhardi deficient in Phosphoribulokinase activity. Plant Physiol 46(4):576. doi:10.1104/Pp.46.4.576

Pick TR, Brautigam A, Schulz MA, Obata T, Fernie AR, Weber APM (2013) PLGG1, a plastidic glycolate glycerate transporter, is required for photorespiration and defines a unique class of metabolite transporters. Proc Natl Acad Sci U S A 110(8):3185-3190. doi:10.1073/pnas.1215142110

Reumann S (2004) Specification of the peroxisome targeting signals type 1 and type 2 of plant peroxisomes by bioinformatics analyses. Plant Physiol 135 (2):783-800. doi:10.1104/pp.103.035584

Reumann S, Weber AP (2006) Plant peroxisomes respire in the light: some gaps of the photorespiratory C2 cycle have become filled-others remain. Biochim Biophys Acta 1763(12):1496-1510. doi:10.1016/j.bbamcr.2006.09.008

Segre D, Deluna A, Church GM, Kishony R (2005) Modular epistasis in yeast metabolism. Nat Genet 37(1):77-83. doi:10.1038/ng1489

Sicher RC, Bunce JA (1997) Relationship of photosynthetic acclimation tochanges of Rubisco activity in field-grown winter wheat and barley during growth in elevated carbon dioxide. Photosynth Res 52:27-38. doi:10.1023/ A:1005874932233

Somerville CR, Ogren WL (1979) A phosphoglycolate phosphatase-deficient mutant of Arabidopsis. Nature 280:833-836. doi:10.1038/280833a0

Somerville CR, Ogren WL (1986) Inhibition of photosynthesis in Arabidopsis mutants lacking leaf glutamate synthase activity. Nature 286:257-259. doi:10.1038/286257a0

Suthers PF, Zomorrodi A, Maranas CD (2009) Genome-scale gene/reaction essentiality and synthetic lethality analysis. Mol Syst Biol 5. doi:10.1038/ Msb.2009.56

Timm S, Nunes-Nesi A, Pamik T, Morgenthal K, Wienkoop S, Keerberg O, Weckwerth W, Kleczkowski LA, Fernie AR, Bauwe H (2008) A cytosolic pathway for the conversion of Hydroxypyruvate to glycerate during photorespiration in Arabidopsis. Plant Cell 20(10):2848-2859. doi:10.1105/ tpc.108.062265

Voll LM, Jamai A, Renne P, Voll H, McClung CR, Weber APM (2006) The photorespiratory Arabidopsis shm1 mutant is deficient in SHM1. Plant Physiol 140(1):59-66. doi:10.1104/pp.105.071399

Wang CL, Guo LY, Li YX, Wang Z (2012) Systematic comparison of C3 and C4 plants based on metabolic network analysis. Bmc Syst Biol 6. doi:10.1186/ 1752-0509-6-S2-S9

Wingler A, Lea PJ, Leegood RC (1997) Control of photosynthesis in barley plants with reduced activities of glycine decarboxylase. Planta 202(2):171-178. doi:10.1007/s004250050116

Wingler A, Ann VJ, Lea PJ, Leegood RC (1999) Serine: glyoxylate aminotransferase exerts no control on photosynthesis. J Exp Bot 50(334):719-722. doi:10.1093/ $\mathrm{jxb} / 50.334 .719$ 
Wingler A, Lea PJ, Quick WP, Leegood RC (2000) Photorespiration: metabolic pathways and their role in stress protection. Philos T R Soc B 355(1402):1517-1529. doi:10.1098/rstb.2000.0712

Zelitch I, Schultes NP, Peterson RB, Brown P, Brutnell TP (2009) High glycolate oxidase activity is required for survival of maize in normal Air. Plant Physiol 149(1):195-204. doi:10.1104/pp.108.128439

doi:10.1186/1939-8433-6-20

Cite this article as: Lakshmanan et al:: Identifying essential genes/

reactions of the rice photorespiration by in silico model-based analysis.

Rice 2013 6:20.

Submit your manuscript to a SpringerOpen ${ }^{\circ}$ journal and benefit from:

- Convenient online submission

- Rigorous peer review

- Immediate publication on acceptance

- Open access: articles freely available online

- High visibility within the field

- Retaining the copyright to your article

Submit your next manuscript at $\boldsymbol{\nabla}$ springeropen.com 